\title{
On Fuzzy Statistical (0)-Convergence in Fuzzy Riesz Spaces
}

\author{
Shatha Abdul-Hussein Kadhim
}

Department of Mathematics, Collage of Sciences, University of Basrah, Basrah, Iraq

\begin{tabular}{ll}
\hline \multicolumn{1}{c}{ Articles Information } & \multicolumn{1}{c}{ Abstract } \\
\hline Received: & In this paper, we present the concepts of fuzzy order convergence, fuzzy statistical (O)- \\
01.04 .2020 & Convergence (Fso-Convergence), and fuzzy statistical Cauchy sequence in fuzzy Riesz \\
Accepted: & spaces, their fundamental properties are established, some important theorems are given, and \\
26.08 .2020 & show every (Fso-Convergence) can be fuzzy statistical Cauchy and prove that any one of two \\
Published: & sequences is fuzzy order converge leads to the second sequence is fuzzy statistical (O)- \\
26.09 .2020 & Convergence. In addition, we have also demonstrated through examples that, in general, \\
Keywords: & "fuzzy ordered linear space, need not to be a fuzzy Riesz spaces". \\
Fuzzy Riesz spaces & \\
Fuzzy order convergence & \\
Fuzzy statistical (O)-convergence & \\
Fuzzy statistical Cauchy sequence & \\
\hline
\end{tabular}

DOI: 10.22401/ANJS.23.3.08

Corresponding author: shatha.alkhaqani@gmail.com

\section{Introduction}

The hypothesis of, vector lattices (Riesz spaces) was founded, separately, by F. Riesz, H. Freudenthal and L. V. Kantorovitch in the Years around (1935), and it is stimulating to perceive. So several authors have developed the subject in [1]. In 1967, the basic concepts in the study of Riesz spaces are the convergence in [2].

So in (1951), Fast [3] and Steinhaus [4], introduced the idea of (Statistical Convergence for Sequences), as a result the statistical convergence has several applications indifferent fields of mathematics some of these fields, including topology [5,6] and fuzzy analysis [7, 8, 9, 10]. So separately from some basic, and main properties of this notion was studied by, buck [11], Salàt [12], Shoenberg [13].The concept of "fuzzy norm" was presented by Katsaras [14]. In reading (Fuzzy Topological Vector Spaces). Consequently [15,16] discussed several topological properties of fuzzy normed linear space. In (1992), employ the connotation of fuzzy norm., Felbin [17], Put progression the fuzzy no.rm on a line.ar space, which is based on their formation provided of a fuzzy metric introduced into [18, 19].In (1995), Beg and Islam introduced the conception fuzzy ordered linear spaces in [20], also in 1994 they are deliberate notion of fuzzy Riesz spaces (vector lattices) [21]. Then many other authors have developed the subject. One of these Labuschagne, Pinchuck in [22].

Following the statistical convergence for sequence was further information in variously spaces. One of these spaces that is fuzzy Riesz space thus we introduce fuzzy statistical (O)-Convergence in, fuzzy Riesz Space. In this framework, established basic definitions and procure several basic results. Also explained that a fuzzy ordered line.ar space not necessity be a (fuzzy Riesz space) in two examples.

\section{Preliminaries}

In this section, we recall some of the basic concepts related to the notions of fuzzy statistical $(\mathrm{O})$-Convergence (FsoConvergence) in fuzzy vector lattices which we will use throughout the paper, and present examples on fuzzy Riesz spaces.

Let $X$ be a universal set and, $A$ be a subset of $X$.

Definition 2.1 [20]. A fuzzy order, $A$ is a fuzzy subset of $X \times X$ such that the conditions are satisfied.

i. For every $x \in X, \dot{\mu}(x, x)=1$ (reflexivity).

ii. For all, $x, y \in X \dot{\mu}(x, y)+\dot{\mu}(y, x)>1$ implies $x=y$ (antisymmetry).

iii. For each $x, y, z \in X$

$$
\dot{\mu}(x, z) \geq \bigvee_{y \in X}[\dot{\mu}(x, y) \wedge \dot{\mu}(y, z)]
$$

where $\dot{\mu}: X \times X \longrightarrow[0,1]$ is the membership function of the fuzzy subset of $X \times X$.

A set with a fuzzy order defined on is called a Fuzzy Ordered Set "foset, for short".

Proposition 2.2 [24]. A subset $A$ of a fuzzy ordered set $X$.

i. $\sup A$, if it exists is unique,

ii. inf $A$, if it exists is unique. 


\section{Al-Nahrain Journal of Science}

ANJS, Vol.23 (3), September, 2020, pp. $61-67$

Proposition 2.3 [24]. In any foset $X$. The following identities hold:

i. $x \wedge x=x, \mathrm{X} \vee \mathrm{X}=\mathrm{x}$, (idempotent),

ii. $x \wedge y=y \wedge x, \mathrm{x} \vee y=\mathrm{y} \vee \mathrm{x}$, (commutative),

iii. $x \wedge(x \vee y)=\mathrm{x} \vee(x \wedge \mathrm{y})=\mathrm{x}$, (absorption),

iv. $\dot{\mu}(x, y)>\frac{1}{2}$, if and only if $x \wedge y=x$ if and only if, $\mathrm{x} \vee y=\mathrm{y}$ (consistency).

Definition 2.4 [20]. A (real) linear space $X$ is said to be a fuzzy ordered linear space if $X$ is a foset and further $X$ satisfies the following conditions.

i. If $x_{1}, x_{2} \in X$, such that, $\dot{\mu}\left(x_{1}, x_{2}\right)>\frac{1}{2}$, then, $\dot{\mu}\left(x_{1}, x_{2}\right) \leq \dot{\mu}\left(x_{1}+x, x_{2}+x\right)$, for all $x \in X$,

ii. If $x_{1}, x_{2} \in X$, such that, $\dot{\mu}\left(x_{1}, x_{2}\right)>\frac{1}{2}$, then $\dot{\mu}\left(x_{1}, x_{2}\right) \leq \dot{\mu}\left(\alpha x_{1}, \alpha x_{2}\right)$, for all $0 \leq \alpha \in \mathbb{R}$.

Definition 2.5 [24]. A foset $X$, is called a Fuzzy Lattice if every finite subsets of $X$ has a supremum, and infimum. So fuzzy lattice $X$ is called Complete if every subset of $X$, has a supremum in addition to, infimum.

Definition 2.6[4] Fuzzy ordered linear space, at the same time is also "fuzzy lattice" is called a (fuzzy Riesz space). Thus fuzzy ordered linear space need not be a fuzzy Riesz space.

As it can be seen in the following two examples:

Example 2.7. In $X=P[0,1]$ the set of all polynomials is a linear space defined over $[0,1]$ with pointwise ordering. Define a function, $\dot{\mu}: X \times X \rightarrow[0,1]$ by, $\dot{\mu}(f, g)=$ 1 when $f(x)=g(x), \dot{\mu}(f, g)=\frac{3}{4}, \quad$ if $\quad f(x) \leq g(x)$, for all $x \in[0,1]$ and $f(x) \neq g(x), \quad$ and $\quad \dot{\mu}(f, g)=0$, otherwise.

Now $(X, \dot{\mu})$, is not a "fuzzy Riesz space" because, the supremum is not a polynomial.

Example 2.8. Let $X=\mathbb{R}^{2}$ be the two-dimensional real space with the coordinate wise ordering.

Define the function $\dot{\mu}: X \times X \rightarrow[0,1]$, by:

$$
\dot{\mu}(x, y)= \begin{cases}1, & x=y, \\ \frac{3}{4}, & x_{1} \leq y_{1}, x_{2} \leq y_{2} \text { and } x \neq y, \\ \frac{2}{3}, & x_{1}<y_{1} \text { and } x_{2}>y_{2}, \\ 0, & \text { otherwise }\end{cases}
$$

Observe that $X$ equipped with $\dot{\mu}$ is a "fuzzy ordered space", however the closed unit disc,

$$
U=\left\{\left(x_{1}, x_{2}\right) \in \mathbb{R}^{2}: x_{1}^{2}+x_{2}^{2} \leq 1\right\}
$$

equipped with the same coordinate wise ordering is not fuzzy Riesz space.

To see this, consider $(1,0),(1,0) \in U$. We see that every upper bound of $\{(1,0),(1,0)\}$ must be greater than or equal to 1 in both it's coordinates.
Therefore $U$, does not contain an upper bound of $\{(1,0),(1,0)\}$ and thus it does not contain it's supremum.

Definition 2.9 [21]. Let $x$ be an element of $X$, then $x_{+}=x \vee 0$ (positive part of $x$ ), and $x_{-}=(-x) \vee 0$ (the negative part) also, $|x|=x \vee(-x)$ (absolute value of $x$ ).

Proposition 2.10 [21]. For any $x \in X$, the elements $x_{+}$, $x_{-}$and $|x|$ are positive. Also the following equalities hold,

i. $x=x_{+}-x_{-}$.

ii. $|x|=x_{+}+x_{-}$.

Theorem 2.11 [21]. In a fuzzy Riesz space, the absolute value has the following properties:
i. $\dot{\mu}(|x+y|,|x|+|y|)>\frac{1}{2}$,
ii. $|\alpha x|=|\alpha||x|$,
iii. $\dot{\mu}(|| x|-| y||,|x-y|)>\frac{1}{2}$,
iv. $|x-y|=(x \vee y)-(x \wedge y)$.

Definition 2.12 [21]. Let $X$ be a fuzzy Riesz space. Two elements $x_{1}, x_{2} \in X$ are said to be orthogonal if $\left|x_{1}\right| \wedge\left|x_{2}\right|=0$, and are written as $x_{1} \perp x_{2}$.

The definition can be extended to the subsets of $X$. Two subsets $A_{1}$ and $A_{2}$ are said to be, Orthogonal if $x_{1} \perp x_{2}$, for any $x_{1} \in A_{1}$, and $x_{2} \in A_{2}$.

Definition 2.13. A sequence $\left(p_{j}\right)_{j \in \mathbb{N}}$, in fuzzy Riesz space is called $((\mathrm{O})$-Sequence $)$ if and only if $\left(p_{j_{i}}\right)_{i \in \mathbb{N}}$ is a decreasing subsequence as a result $p_{j_{i}} \downarrow_{F s t} 0$ and $0=\inf _{i \in \mathbb{N}} p_{j_{i}}$.

Definition 2.14. A sequence $\left(x_{j}\right)_{j \in \mathbb{N}}$ in a fuzzy Riesz space $X$ is said to be fuzzy order convergence to an element $x_{0} \in X$, denoted, " $x_{j} \stackrel{F_{0}}{\longrightarrow} x_{0}$ " if there exists another sequence, $\left(p_{j}\right)_{j \in \mathbb{N}}$, such that $p_{j} \downarrow_{F u z} 0$, such that for every $j \in \mathbb{N}$ with the Property that $\dot{\mu}\left(\left|x_{j}-x_{0}\right|, p_{j}\right)>\frac{1}{2}$. In this case, $x_{0}$ is called the Fuzzy Order Limit of $\left(x_{j}\right)_{j \in \mathbb{N}}$.

Notation 2.15. Let $F$ be a set of positive integers. The natural density of $F$ is distinct as:

$$
\delta(F)=\lim _{n \longrightarrow \infty} \frac{1}{n}\left|\left\{f \in F: \dot{\mu}(f, n)>\frac{1}{2}\right\}\right|
$$




\section{Al-Nahrain Journal of Science}

ANJS, Vol.23 (3), September, 2020, pp. $61-67$

where $\left|\left\{f \in F: \dot{\mu}(f, n)>\frac{1}{2}\right\}\right|$ denotes the number of elements $F$ not more than $n$.

If $\delta(F)$ exists, Then $\delta\left(F^{\circ}\right)=1-\delta(F)$, thus $F^{c}$ is the complement of the set, $F$ in $\mathbb{N}$.

\section{Main results}

In this section, We submitted a new concept, namely fuzzy statistically (O)-convergent (Fso-Convergence) defined as follows. So we established some properties and prove an important theorems related to this concept.

Definition 3.1. Let $X$ be a fuzzy Riesz space. A sequence $\left(x_{j}\right)_{j \in \mathbb{N}}$ of elements of $X$ is fuzzy statistically increasing if there exists a set $F=\left\{j_{1}<j_{2}<\ldots\right\} \subset \mathbb{N}$, such that $\delta(F)=1$ and $\left(x_{j_{i}}\right)_{i \in \mathbb{N}}$ is increasing with the aim of if $j_{i} \leq j_{i+1}$ implies that $\dot{\mu}\left(x_{j_{i}}, x_{j_{i+1}}\right)>\frac{1}{2}$. In this case, we note down $x_{j} \uparrow^{F_{s t}}$.If moreover the element $x_{0}=\sup _{i \in \mathbb{N}} x_{j_{i}}$ exists, then we note down $x_{j} \uparrow^{\text {Fst }} x_{0}$.

A fuzzy statistically decreasing sequence is defined similarly, then can write, $x_{j} \downarrow_{F_{s t}}$. Also if $x_{0}=\inf _{i \in \mathbb{N}} x_{j_{i}}$ exists, then $x_{j} \downarrow_{F s t} x_{0}$.

Definition 3.2. Let $X$ be a fuzzy Riesz space. A sequence $\left(x_{j}\right)_{j \in \mathbb{N}}$ in $X$ is fuzzy statistically (O)-Convergent to an element $x_{0} \in X$. If there exists $(\mathrm{O})$-Sequence $\left(p_{j}\right)_{j \in \mathbb{N}}$ and a set $F=\left\{j_{1}<j_{2}<\ldots\right\}$ of $\mathbb{N}$ with $\delta(F)=1$ such that, $\left\{j \in \mathbb{N}: \dot{\mu}\left(p_{j},\left|x_{j}-x_{0}\right|\right)>\frac{1}{2}\right\}$ has natural density zero, in this case, we write $x_{j} \stackrel{\text { Fso }}{\longrightarrow} x_{0}$ and $x_{0}$ is called the Fso-limit of the sequence $\left(x_{j}\right)_{j \in \mathbb{N}}$.

Definition 3.3. Let $X$ be a fuzzy Riesz space. A sequence $\left(x_{j}\right)_{j \in \mathbb{N}}$ in $X$ is fuzzy statistically Cauchy sequence if there exists (O)-Sequence $\left(p_{j}\right)_{j \in \mathbb{N}}$ a subset $F=\left\{j_{i}<j_{i}<\ldots\right\}$ of $\mathbb{N}$, with $\delta(F)=1$ and there exists, number $k=k(\epsilon)$ such that, $\left\{j \in \mathbb{N}: \dot{\mu}\left(p_{j},\left|x_{j}-x_{k(\Theta)}\right|\right)>\frac{1}{2}\right\}$ has natural density zero.

Theorem 3.4. Let $X$ be a fuzzy Riesz space, and $\left(x_{j}\right)_{j \in \mathbb{N}}$ be a, sequence of elements in $X$. Then we have,

i. If $\left(x_{j}\right)_{j \in \mathbb{N}}$ is fuzzy statistically increasing, then the set, $\left\{j \in \mathbb{N}: \dot{\mu}\left(x_{j+1}, x_{j}\right)>\frac{1}{2}\right\}$ has natural density zero. ii. If $\left(x_{j}\right)_{j \in \mathbb{N}}$ is fuzzy statistically decreasing, then the set $\left\{j \in \mathbb{N}: \dot{\mu}\left(x_{j}, x_{j+1}\right)>\frac{1}{2}\right\}$. has natural density zero.

Proof.

i. Let $\left(x_{j}\right)_{j \in \mathbb{N}}$ be a fuzzy statistically increasing sequence in $X$, then there exists a set $F=\left\{j_{1}<j_{2}<\ldots\right\} \subset \mathbb{N}$, such that $\delta(F)=1$ and $\left(x_{j_{i}}\right)_{i \in \mathbb{N}}$ is increasing. Now since $\left\{j \in F: \dot{\mu}\left(x_{j}, x_{j+1}\right)>\frac{1}{2}\right\} \subset\left\{j \in \mathbb{N}: \dot{\mu}\left(x_{j}, x_{j+1}\right)>\frac{1}{2}\right\}$ hence we have the set, $\left\{j \in \mathbb{N}: \dot{\mu}\left(x_{j+1}, x_{j}\right)>\frac{1}{2}\right\}$ has natural density zero.

ii. This part can be proved similarly.

Theorem 3.5. Let $\left(x_{j}\right)_{j \in \mathbb{N}}$ and $\left(y_{j}\right)_{j \in \mathbb{N}}$ be Sequence in fuzzy Riesz space $X$. Then the following satisfies:

i. If $x_{j} \uparrow^{F_{s t}} x_{0}, x_{j} \downarrow_{F_{s t}} x_{0}$ and $\alpha \geq 0$, then $\alpha x_{j} \uparrow^{F_{s t}} \alpha x_{0}$ and $\alpha x_{j} \downarrow_{F s t} \alpha x_{0} \cdot$

ii. If $x_{j} \downarrow_{F s t} 0$ and $y_{j} \downarrow_{F s t} 0$, then $x_{j}+y_{j} \downarrow_{F s t} 0$.

Proof.

i. This is obvious. From definition.

ii. Suppose that $x_{j} \downarrow_{F s t} 0$ and $y_{j} \downarrow_{F s t} 0$, then we define the following sets:

$$
\begin{aligned}
& F=\left\{j_{1}<j_{2}<\ldots\right\} \subset \mathbb{N}, \\
& H=\left\{k_{:}<k_{2}<\ldots\right\} \subset \mathbb{N}
\end{aligned}
$$

such that $\delta(F)=\delta(H)=1$, also let $\left(x_{j_{i}}\right)_{i \in \mathbb{N}}$ and $\left(y_{k_{i}}\right)_{i \in \mathbb{N}}$ are a subsequence of $\left(x_{j}\right)_{j \in \mathbb{N}}$ and $\left(y_{j}\right)_{j \in \mathbb{N}}$ (respectively). Evidently (0) is an lower bound of the subsequences. Now assume that the set $F \cap H=\left\{r_{1}<r_{2}<\ldots\right\} \subset \mathbb{N}$ with $\delta(F \cap H)=1$. One infers that the sequence $\dot{\mu}\left(0, x_{r_{i}}+y_{r_{i}}\right)>\frac{1}{2}$ for every $i \in \mathbb{N}$. Hence $0 \in \mathrm{L}\left(\left\{x_{r_{t}}+y_{r_{t}}: i \in \mathbb{N}\right\}\right)$. Now let $p$ is another lower bound of this set, it follows that $\dot{\mu}\left(p, x_{r}+y_{r_{i}}\right)>\frac{1}{2}$ for every $i \in \mathbb{N}$. Thus $r \leq r_{i}$, where a member $r$ of the index set $F \cap H$. From this we write, $\quad \dot{\mu}\left(p, x_{r_{i}}+y_{r_{i}}\right)>\frac{1}{2} \quad$ and $\dot{\mu}\left(x_{r_{i}}+y_{r_{i}}, x_{r}+y_{r_{i}}\right)>\frac{1}{2}$. Therefore by transitivity $\dot{\mu}\left(p, x_{r}+y_{r_{i}}\right)>\frac{1}{2}$ for every $r \leq r_{i}$. It implies that $p \in \mathrm{L}\left(\left\{x_{r}+y_{r_{t}}: i \in \mathbb{N}\right\}\right)$. It further implies that $p \in \mathbf{L}\left(\left\{\inf _{r, r_{t} \in \mathbb{N}}\left(x_{r}+y_{r_{t}}\right): i \in \mathbb{N}\right\}\right)$ $p \in \mathrm{L}\left(\left\{\left(x_{r}\right): r \in F \cap H\right\}\right) \quad$ means that 


\section{Al-Nahrain Journal of Science}

ANJS, Vol.23 (3), September, 2020, pp. $61-67$

\footnotetext{
$\dot{\mu}\left(p, x_{r}\right)>\frac{1}{2}$. One infers that for every $r \in F \cap H$ we have $\dot{\mu}\left(p, \inf _{r \in F \cap H} x_{r}\right)>\frac{1}{2}$ means that $\dot{\mu}(p, 0)>\frac{1}{2}$. Thus, $\inf _{i \in \mathbb{N}}\left(x_{r_{i}}+y_{r_{i}}\right)=0$, which shows that $x_{j}+y_{j} \downarrow_{F s t} 0$.
}

Theorem 3.6. Let $X$ be a fuzzy Riesz spaces, $\left(x_{j}\right)_{j \in \mathbb{N}}$ and $\left(y_{j}\right)_{j \in \mathbb{N}}$ are two sequences in $X$. Then:

i. If $\left(x_{j}\right)_{j \in \mathbb{N}}$ be a Fso-Convergence to both $x_{0}$ and $y_{0}$ then $x_{0}=y_{0}$.

ii. If $x_{j} \stackrel{\text { Fso }}{\longrightarrow} x_{0}$ and $y_{j} \stackrel{\text { Fso }}{\longrightarrow} y_{0}$, then $\alpha x_{j} \stackrel{\text { Fso }}{\longrightarrow} \alpha x_{0}, \alpha \in \mathbb{R} \quad$ and $x_{j}+y_{j} \stackrel{\text { Fso }}{\longrightarrow} x_{0}+y_{0}$.

iii. If $x_{j} \stackrel{F s o}{\longrightarrow} x_{0}$ and $y_{j} \stackrel{F s o}{\longrightarrow} y_{0} \quad$, then $x_{j} \vee y_{j} \stackrel{\text { Fso }}{\longrightarrow} x_{0} \vee y_{0} \quad$ and, $x_{j} \wedge y_{j} \stackrel{\text { Fso }}{\longrightarrow} x_{0} \wedge y_{0}$.

iv. If $\quad x_{j} \stackrel{\text { Fso }}{\longrightarrow} x_{0}$ then $x_{j}^{+} \stackrel{\text { Fso }}{\longrightarrow} x_{0}^{+}$, $x_{j}^{-} \stackrel{\text { Fso }}{\longrightarrow} x_{0}^{-}$, and $\left|x_{j}\right| \stackrel{\text { Fso }}{\longrightarrow}\left|x_{0}\right|$.

v. If $x_{j} \stackrel{F s o}{\longrightarrow} x_{0}$ and $\dot{\mu}\left(y_{j}, x_{j}\right)>\frac{1}{2}$, then $\dot{\mu}\left(y_{0}, x_{0}\right)>\frac{1}{2}$.

\section{Proof.}

i. By Definition $3.2 x_{j} \stackrel{F_{50}}{\longrightarrow} x_{0}$, reveal there exists (O)-Sequences $\left(p_{j_{i}}\right)_{i \in \mathbb{N}}$ and $\left(q_{j_{i}}\right)_{i \in \mathbb{N}}$ in $X$, and a set $F_{1}=\left\{j_{1}<j_{2}<\ldots\right\} \subset \mathbb{N}$, with $\delta(F)=1$ so as to $\dot{\mu}\left(\left|x_{j_{i}}-x_{0}\right|, p_{j_{i}}\right)>\frac{1}{2}, \dot{\mu}\left(\left|z_{j_{i}}-x_{0}\right|, q_{j_{i}}\right)>\frac{1}{2}$ for every $i \in \mathbb{N}$. So we know $\dot{\mu}\left(0,\left|x_{0}-y_{0}\right|\right)>\frac{1}{2}$. Thereby, we have that:

$$
\dot{\mu}\left(\left|x_{0}-y_{0}\right|,\left|x_{j_{i}}-x_{0}\right|+\left|x_{j_{i}}-y_{0}\right|\right)>\frac{1}{2}
$$

and,

$$
\dot{\mu}\left(\left|x_{j_{i}}-x_{0}\right|+\left|x_{j_{i}}-y_{0}\right|, p_{j_{i}}+q_{j_{i}}\right)>\frac{1}{2}
$$

for every $i \in \mathbb{N}$. For that reason by transitivity $\dot{\mu}\left(\left|x_{0}-y_{0}\right|, p_{j_{i}}+q_{j_{i}}\right)>\frac{1}{2}$ for every $i \in \mathbb{N}$. Hence we have $x_{0}=y_{0}$.

ii. By definition $x_{j} \stackrel{\text { Fso }}{\longrightarrow} x_{0}$ implies, there exists (O)sequence $\left(p_{j}\right)_{j \in \mathbb{N}}$. Supplementary the set $\left\{j \in \mathbb{N}: \dot{\mu}\left(p_{j},\left|x_{j}-x_{0}\right|\right)>\frac{1}{2}\right\}$ has natural density zero. Firstly, let $\alpha>0$, since $\left(p_{j}\right)_{j \in \mathbb{N}}$ be (O)sequence, then $p_{j_{i}} \downarrow_{F_{s t}} 0$ and $0=\inf _{i \in \mathbb{N}} p_{j_{i}}$ implies that $\alpha p_{j_{i}} \downarrow_{F_{s t}} 0$ and $0=\inf _{i \in \mathbb{N}} \alpha p_{j_{i}}$. Thus, the set $\left\{j \in \mathbb{N}: \dot{\mu}\left(\alpha p_{j}, \alpha\left|x_{j}-x_{0}\right|\right)>\frac{1}{2}\right\}$ has natural density zero. Hence we have, $\alpha x_{j} \stackrel{F_{s 0}}{\longrightarrow} \alpha x_{0}$.

Now if $\alpha<0$, would imply the (O)-Sequence $\left(p_{j}\right)_{j \in \mathbb{X}}$ be increasing means that $\alpha p_{j_{i}} \uparrow^{F_{s t}} 0$, then in this case, the definition, Fso-Convergent is not satisfy.

Now Let another Sequence in $X,\left(y_{j}\right)_{j \in \mathbb{N}}$ such that $y_{j} \stackrel{F s o}{\longrightarrow} y_{0}$. Then there exists (O)-Sequence $\left(p_{j}\right)_{j \in \mathbb{N}}$ and $\left(q_{j}\right)_{j \in \mathbb{N}} \quad$ such that the sets $\left\{j \in \mathbb{N}: \dot{\mu}\left(p_{j},\left|x_{j}-x_{0}\right|\right)>\frac{1}{2}\right\} \quad$ and $\left\{j \in \mathbb{N}: \dot{\mu}\left(q_{j},\left|y_{j}-y_{0}\right|\right)>\frac{1}{2}\right\}$ has natural density zero. Consequently, $\dot{\mu}\left(\left|\left(x_{j}+y_{j}\right)-\left(x_{0}+y_{0}\right)\right|, \mid\left(x_{j}+\right.\right.$ $\left.\left.y_{j}\right)-\left(x_{0}+y_{j}\right)+\left(x_{0}+y_{j}\right)-\left(x_{0}+y_{0}\right) \mid\right)>\frac{1}{2} \quad$, implies that $\dot{\mu}\left(\mid\left(x_{j}+y_{j}\right)-\left(x_{0}+y_{j}\right)+\left(x_{0}+y_{j}\right)-\right.$ $\left.\left(x_{0}+y_{0}\right)|,| x_{j}-x_{0}|+| y_{j}-y_{0} \mid\right)>\frac{1}{2}$, for every $j \in \mathbb{N}$. By transitivity we have for every $j \in \mathbb{N}, \dot{\mu}\left(\mid\left(x_{j}+\right.\right.$ $\left.\left.y_{j}\right)-\left(x_{0}+y_{0}\right)|,| x_{j}-x_{0}|+| y_{j}-y_{0} \mid\right)>\frac{1}{2}$. Clearly, that $\dot{\mu}\left(\left|x_{j}-x_{0}\right|+\left|y_{j}-y_{0}\right|, p_{j}+q_{j}\right)>\frac{1}{2}$ for every $j \in \mathbb{N}$ and transitivity yields $\dot{\mu}\left(\left(x_{j}+y_{j}\right)-\left(x_{0}+y_{0}\right) \mid, p_{j}+q_{j}\right)>\frac{1}{2}$ where $\left(p_{j}+q_{j}\right)_{j \in \mathbb{N}}$ be O-Sequence because $p_{j_{i}}+q_{j_{i}} \downarrow_{F_{s t}} 0 \quad$ and $\inf _{i \in \mathbb{N}}\left(p_{j_{i}}+q_{j_{i}}\right)=\inf _{i \in \mathbb{N}} p_{j_{i}}+\inf _{i \in \mathbb{N}} q_{j_{i}}=0$. Hence, can have the, $x_{j}+y_{j} \stackrel{\text { Fso }}{\longrightarrow} x_{0}+y_{0}$.

iii. Since $x_{j} \stackrel{F s o}{\longrightarrow} x_{0}$, and $\mathrm{y}_{j} \stackrel{\text { Fso }}{\longrightarrow} y_{0}$, there exist two (O)-Sequences $\left(p_{j}\right)_{j \in \mathbb{N}}$ and, $\left(q_{j}\right)_{j \in \mathbb{N}}$ such that, $\dot{\mu}\left(\left|x_{j}-x_{0}\right|, p_{j}\right)>\frac{1}{2}$ and $\dot{\mu}\left(\left|y_{j}-y_{0}\right|, q_{j}\right)>\frac{1}{2}$, for every $j \in \mathbb{N}$. Consequently $\dot{\mu}\left(\left|\left(x_{j} \vee y_{j}\right)-\left(x_{0} \vee y_{0}\right)\right|, \mid x_{j}-\right.$ $\left.x_{0}|+| y_{j}-y_{0} \mid\right)>\frac{1}{2}$, for every $j \in \mathbb{N}$.

$$
\begin{aligned}
& \text { Also clearly: } \\
& \dot{\mu}\left(\left|x_{j}-x_{0}\right|+\left|y_{j}-y_{0}\right|, p_{j}+q_{j}\right)>\frac{1}{2}
\end{aligned}
$$

Therefore by transitivity we have:

$$
\dot{\mu}\left(\left|\left(x_{j} \vee y_{j}\right)-\left(x_{0} \vee y_{0}\right)\right|, p_{j}+q_{j}\right)>\frac{1}{2}
$$

for every $j \in \mathbb{N}$. From prove the part (ii), we have $\left(p_{j}+q_{j}\right)_{j \in \mathbb{N}}$ be O-Sequence. Thus, $x_{j} \vee y_{j} \stackrel{\text { Fso }}{\longrightarrow} x_{0} \vee y_{0}$.

Similarly, we have that $x_{j} \wedge y_{j} \stackrel{\text { Fso }}{\longrightarrow} x_{0} \wedge y_{0}$. 


\section{Al-Nahrain Journal of Science}

ANJS, Vol.23 (3), September, 2020, pp. $61-67$

iv. Let $\left(x_{j}\right)_{j \in \mathbb{N}}$ be a sequence in $X$ thus $x_{j} \stackrel{F s o}{\longrightarrow} x_{0}$, then there exist (O)-Sequence, $\left(p_{j}\right)_{j \in \mathbb{N}}$ of element in $X$ and a set $F_{1}=\left\{j_{1}<j_{1}<\ldots\right\} \subset \mathbb{N}$, with $\delta(F)=1$ such that the set, $\left\{j \in \mathbb{N}: \dot{\mu}\left(\left|x_{j}-x_{0}\right|, p_{j}\right)>\frac{1}{2}\right\}$, has natural density 1 . To prove the first assertion, $x_{j}^{+} \stackrel{\text { Fso }}{\longrightarrow} x_{0}^{+}$. Thus we can write $\left\{j \in \mathbb{N}: \dot{\mu}\left(\left|x_{j}-x_{0}\right|, p_{j}\right)>\frac{1}{2}\right\}$ be a subset of the set, $\left\{j \in \mathbb{N}: \dot{\mu}\left(\left|x_{j}^{+}-x_{0}^{+}\right|, p_{j}\right)>\frac{1}{2}\right\}$. Therefore, we have $x_{j}^{+} \stackrel{\text { Fso }}{\longrightarrow} x_{0}^{+}$. Similarly, $x_{j}^{-} \stackrel{F_{s o}}{\longrightarrow} x_{0}^{-}$.

Finally, to see that $\left|x_{j}\right| \stackrel{F s o}{\longrightarrow}\left|x_{0}\right|$ we can write the set, $\left\{j \in \mathbb{N}: \dot{\mu}\left(\left|x_{j}-x_{0}\right|, p_{j}\right)>\frac{1}{2}\right\}$. is a subset of the set $\left\{j \in \mathbb{N}: \dot{\mu}\left(|| x_{j}|-| x_{0}||, p_{j}\right)>\frac{1}{2}\right\} \quad$ where $\left(p_{j}\right)_{j \in \mathbb{N}}$ be O-Sequence. Hence we have that $\left|x_{j}\right| \stackrel{F s o}{\longrightarrow}\left|x_{0}\right| \cdot$

v. Suppose, $x_{j} \stackrel{F s o}{\longrightarrow} x_{0}$ and $\dot{\mu}\left(x_{j}, 0\right)>\frac{1}{2}$ for every $j \in \mathbb{N}$. From this has $x_{j}^{-}=\left(-x_{j}\right) \vee 0=0$. It further implies that $-x_{j} \in U(0)$ imply that $\dot{\mu}\left(-x_{j}, 0\right)>\frac{1}{2}$ for every $j \in \mathbb{N}$. By using the part (iv), have that $x_{j}^{-} \stackrel{\text { Fso }}{\longrightarrow} x_{0}^{-}$, where $x_{0}^{-}=0$. Thereby, be able to write $\dot{\mu}\left(0, x_{0}\right)>\frac{1}{2}$ Since $x_{j} \stackrel{\text { Fso }}{\longrightarrow} x_{0}$ and $\dot{\mu}\left(y_{0}, x_{j}\right)>\frac{1}{2}$ for every $j \in \mathbb{N}$. Therefore condition (i) of the Definition $2.4, \dot{\mu}\left(0, x_{j}-y_{0}\right)>\frac{1}{2}$ for every $j \in \mathbb{N}$. Consequently we can write $x_{j}-y_{0} \stackrel{\text { Fso }}{\longrightarrow} x_{0}-y_{0}$, by using part (ii). Therefore we see that $\dot{\mu}\left(0, x_{0}-y_{0}\right)>\frac{1}{2}$. Hence $\dot{\mu}\left(y_{0}, x_{0}\right)>\frac{1}{2}$.

Theorem 3.7. Let $X$ be a fuzzy Riesz space, $\left(x_{j}\right)_{j \in \mathbb{N}}$, $\left(y_{j}\right)_{j \in \mathbb{N}}$ and $\left(z_{j}\right)_{j \in \mathbb{N}}$ be three sequences of points in $X$, such that:

i. $\dot{\mu}\left(x_{j}, y_{j}\right)>\frac{1}{2}$, and $\dot{\mu}\left(y_{j}, z_{j}\right)>\frac{1}{2}$, for all $\mathrm{j} \in F \subset \mathbb{N}$ with $\delta(F)=1$.

ii. If $x_{j} \stackrel{F s o}{\longrightarrow} x_{0}$ and $z_{j} \stackrel{F s o}{\longrightarrow} x_{0} \quad$, then $\mathrm{y}_{j} \stackrel{F_{s o}}{\longrightarrow} x_{0}$.
Proof. Assume that the given Conditions (i) and (ii) hold for the sequences $\left(x_{j}\right)_{j \in \mathbb{N}},\left(y_{j}\right)_{j \in \mathbb{N}}$ and $\left(z_{j}\right)_{j \in \mathbb{N}}$ suppose two (O)-Sequences $\left(p_{j}\right)_{j \in \mathbb{N}}$ and $\left(q_{j}\right)_{j \in \mathbb{N}}$. Also from condition (ii), have $\delta\left(F_{1}\right)=\delta\left(F_{2}\right)=1$, where $F_{1}=\left\{j_{1}<j_{2}<\ldots\right\} \subset \mathbb{N}, F_{2}=\left\{l_{1}<l_{2}<\ldots\right\} \subset \mathbb{N}$, such that $\dot{\mu}\left(p_{j},\left|x_{j}-x_{0}\right|\right)>\frac{1}{2}$ and $\dot{\mu}\left(q_{j},\left|z_{j}-x_{0}\right|\right)>\frac{1}{2}$ for every $j \in$ $\mathbb{N}$. Also get $\delta\left(F_{1} \cap F_{2}\right)=1$, and from (i) we have $\dot{\mu}\left(x_{j}-x_{0}, y_{j}-x_{0}\right)>\frac{1}{2}$ and $\dot{\mu}\left(y_{j}-x_{0}, z_{j}-x_{0}\right)>\frac{1}{2}$ for every $j \in \mathbb{N}$. This implies:

$$
\dot{\mu}\left(y_{j}-x_{0},\left|x_{j}-x_{0}\right|+\left|z_{j}-x_{0}\right|\right)>\frac{1}{2}
$$

and

$$
\dot{\mu}\left(\left|x_{j}-x_{0}\right|+\left|z_{j}-x_{0}\right|, p_{j}+q_{j}\right)>\frac{1}{2}
$$

By transitivity have: $\dot{\mu}\left(y_{j}-x_{0}, p_{j}+q_{j}\right)>\frac{1}{2}$, for every $\mathrm{j} \in F_{1} \cap F_{2} \subset \mathbb{N}$. Since $\left(p_{j}\right)_{j \in \mathbb{N}}$ and $\left(q_{j}\right)_{j \in \mathbb{N}}$ then $p_{j}+q_{j} \downarrow_{F s t} 0$. So, moreover $\inf _{i \in \mathbb{N}}\left(p_{j_{i}}+q_{j_{i}}\right)=\inf _{i \in \mathbb{N}} p_{j_{i}}+\inf _{i \in \mathbb{N}} q_{j_{i}}=0$. Consequently, for $\left(p_{j}+q_{j}\right)_{j \in \mathbb{N}}$ be (O)-Sequence comprise the set $\left\{j \in \mathbb{N}: \dot{\mu}\left(\left|y_{j}-x_{0}\right|, p_{j}+q_{j}\right)>\frac{1}{2}\right\}$ has natural density 1 . Hence $\mathrm{y}_{j} \stackrel{\text { Fso }}{\longrightarrow} x_{0}$.

Theorem 3.8. Let a sequences $\left(x_{j}\right)_{j \in \mathbb{N}}$ and $\left(y_{j}\right)_{j \in \mathbb{N}}$ be fuzzy Riesz Space $X$. If $\left(y_{j}\right)_{j \in \mathbb{N}}$ is fuzzy order convergent sequence, such that $x_{j}=y_{j}$, then $\left(x_{j}\right)_{j \in \mathbb{N}}$ is Fso-Convergent.

Proof. Suppose $\left\{j \in \mathbb{N}: x_{j} \neq y_{j}\right\}$ has natural density zero and a sequence $\left(y_{j}\right)_{j \in \mathbb{N}}$ is a fuzzy order convergent. Subsequently there exists $(\mathrm{O})$-Sequence $\left(p_{j}\right)_{j \in \mathbb{N}}$ and a set $F=\left\{j_{:}<j_{2}<\ldots\right\} \subset \mathbb{N}$, with $\delta(F)=1$, such that:

$$
\begin{aligned}
& \left\{j \in \mathbb{N}: \dot{\mu}\left(p_{j},\left|x_{j}-x_{0}\right|\right)>\frac{1}{2}\right\} \subseteq \\
& \left\{j \in \mathbb{N}: \dot{\mu}\left(p_{j},\left|y_{j}-x_{0}\right|\right)>\frac{1}{2}\right\} \cup\left\{j \in \mathbb{N}: x_{j} \neq y_{j}\right\}
\end{aligned}
$$

This means that,

$$
\begin{aligned}
& \delta\left(\left\{j \in \mathbb{N}: \dot{\mu}\left(p_{j},\left|x_{j}-x_{0}\right|\right)>\frac{1}{2}\right\}\right) \leq \\
& \delta\left(\left\{j \in \mathbb{N}: \dot{\mu}\left(p_{j},\left|y_{j}-x_{0}\right|\right)>\frac{1}{2}\right\}\right)+\delta\left(\left\{j \in \mathbb{N}: x_{j} \neq\right.\right. \\
& \left.\left.y_{j}\right\}\right)
\end{aligned}
$$




\section{Al-Nahrain Journal of Science}

ANJS, Vol.23 (3), September, 2020, pp. $61-67$

While, $\left(y_{j}\right)_{j \in \mathbb{N}}$ be a fuzzy order convergent to element $x_{0}$ in $X$, the set $\left\{j \in \mathbb{N}: \dot{\mu}\left(p_{j},\left|y_{j}-x_{0}\right|\right)>\frac{1}{2}\right\}$ contain finite number of integers. Hence, the set $\left\{j \in \mathbb{N}: \dot{\mu}\left(p_{j},\left|y_{j}-x_{0}\right|\right)>\frac{1}{2}\right\}$ has natural density zero.

From this include, the set $\left\{j \in \mathbb{N}: \dot{\mu}\left(p_{j},\left|x_{j}-x_{0}\right|\right)>\frac{1}{2}\right\}$ has natural density zero. Accordingly $\left(x_{j}\right)_{j \in \mathbb{N}}$ is FsoConvergent.

Theorem 3.9. If a sequence $\left(x_{j}\right)_{j \in \mathbb{N}}$ in fuzzy Riesz space $X$ is Fso-Convergent, then it is fuzzy statistical Cauchy.

Proof. Let $\left(x_{j}\right)_{j \in \mathbb{N}}$ is Fso-Convergent to $x_{0}$ in $X$. Then there exists $(\mathrm{O})$-Sequence $\left(p_{j}\right)_{j \in \mathbb{N}}$ as well as a set $F=\left\{j_{1}<j_{2}<\ldots\right\} \subset \mathbb{N}$, with $\delta(F)=1$, and will write $A=\left\{j \in \mathbb{N}: \dot{\mu}\left(p_{j},\left|x_{j}-x_{0}\right|\right)>\frac{1}{2}\right\}$ has natural density zero. So, be able to choose positive integers $k(\epsilon)$, such that $k(\in) \in A$. Then, we have the sets:

$$
\begin{aligned}
& B=\left\{j \in \mathbb{N}: \dot{\mu}\left(p_{j},\left|x_{k(\epsilon)}-x_{0}\right|\right)>\frac{1}{2}\right\} \\
& C=\left\{j \in \mathbb{N}: \dot{\mu}\left(2 p_{j},\left|x_{j}-x_{k(\varepsilon)}\right|\right)>\frac{1}{2}\right\}
\end{aligned}
$$

while we explain

$$
\begin{aligned}
& \left\{j \in \mathbb{N}: \dot{\mu}\left(2 p_{j},\left|x_{j}-x_{k(\epsilon)}\right|\right)>\frac{1}{2}\right\} \subseteq \\
& \left\{j \in \mathbb{N}: \dot{\mu}\left(p_{j},\left|x_{j}-x_{0}\right|\right)>\frac{1}{2}\right\} \cup \\
& \left\{j \in \mathbb{N}: \dot{\mu}\left(p_{j},\left|x_{k(\epsilon)}-x_{0}\right|\right)>\frac{1}{2}\right\} .
\end{aligned}
$$

Consequently,

$$
\begin{aligned}
& \delta\left(\left\{j \in \mathbb{N}: \dot{\mu}\left(2 p_{j},\left|x_{j}-x_{k(\epsilon)}\right|\right)>\frac{1}{2}\right\}\right) \leq \\
& \delta\left(\left\{j \in \mathbb{N}: \dot{\mu}\left(p_{j},\left|x_{j}-x_{0}\right|\right)>\frac{1}{2}\right\}\right)+ \\
& \delta\left(\left\{j \in \mathbb{N}: \dot{\mu}\left(p_{j}, \mid x_{k(\epsilon)}-x_{0}\right)>\frac{1}{2}\right\}\right)
\end{aligned}
$$

This leads to the set has, $\left\{j \in \mathbb{N}: \dot{\mu}\left(2 p_{j},\left|x_{j}-x_{k(\epsilon)}\right|\right)>\frac{1}{2}\right\}$ natural density zero. After ward $\left(x_{j}\right)_{j \in \mathbb{N}}$ is fuzzy statistical Cauchy.

Theorem 3.10. Let $X$ be a fuzzy Riesz space. A sequence $\left(x_{j}\right)_{j \in \mathbb{N}}$ is Fso- Convergent to $x_{0}$ in $X$, if and only if there exist a (O)-Sequence $\left(p_{j}\right)_{j \in \mathbb{N}}$ a subset $F=\left\{j_{1}<j_{2}<\ldots\right\}$ of $\mathbb{N}$, such that $\delta(F)=1$ and a subsequence $\left(x_{j_{i}}\right)_{i \in \mathbb{N}}$ of $\left(x_{j}\right)_{j \in \mathbb{N}}$, such that $\left(x_{j_{i}}\right)_{i \in \mathbb{N}}$ is fuzzy convergent to $x_{0}$ in $X$ and, $\delta\left(\left\{j \in \mathbb{N}: \dot{\mu}\left(p_{j}, \mid x_{j}-x_{k(\varepsilon)}\right)>\frac{1}{2}\right\}\right)=0$, for every $i \in \mathbb{N}$.

Proof. Let $\left(x_{j}\right)_{j \in \mathbb{N}}$ be a Sequence in $X$, such that $\left(x_{j}\right)_{j \in \mathbb{N}}$ is Fso-Convergent to $x_{0} \in X$. Let $\left(p_{j}\right)_{j \in \mathbb{N}}$ be $(\mathrm{O})$ sequence and arbitrary subset $F=\left\{j_{1}<j_{2}<\ldots\right\}$ of $\mathbb{N}$.

Put $F^{k}=\left\{j_{1}^{k}<j_{2}^{k}<\ldots\right\}, \mathrm{k}=1,2, \ldots .$. such that $\delta\left(F^{k}\right)=1$, we write $G^{(r)}=\left\{j \in \mathbb{N}: \dot{\mu}\left(\left|x_{j}-x_{0}\right|, p_{j}\right)>\frac{1}{2}\right\}$ for any positive integer $r$. Then for each $r$, we have $G^{(r+1)} \subset G^{(r)}$ and $\delta\left(G^{(r)}\right)=1$. Choose $e(1)$ such that $l>e(1)$, then $G^{(1)} \neq \varnothing$, then for every positive integer $l$ such that $e(1) \leq l<e(2)$, choose $x_{j_{i}} \in G^{(r)}$, this means there exist a subset $F^{1}=\left\{j_{1}^{1}<j_{2}^{1}<\ldots\right\}$ of $\mathbb{N}$ such that $\delta\left(F^{1}\right)=1$, the set $\left\{j \in \mathbb{N}: \dot{\mu}\left(\left|x_{j_{t}}-x_{0}\right|, p_{j}\right)>\frac{1}{2}\right\}$, for every $i \in \mathbb{N}$ has natural density 1 .

In general, choose $e(m+1)>e(m)$, choose $x_{j_{s}} \in G^{(m)}$, we have a subset $F^{n}=\left\{j_{1}^{n}<j_{2}^{n}<\ldots\right\}$ of $\mathbb{N}$, where $\mathrm{m}=1,2, \ldots$, such that $\delta\left(F^{m}\right)=1$, and the set,

$\left\{j \in \mathbb{N}: \dot{\mu}\left(\left|x_{j_{t}}-x_{0}\right|, p_{j}\right)>\frac{1}{2}\right\}$, for every $i \in \mathbb{N}$ has natural density 1 .

Hence it follows that the subsequence $\left(x_{j_{i}}\right)_{i \in \mathbb{N}}$ is fuzzy convergent.

Since $\left(p_{j}\right)_{j \in \mathbb{N}}$ be a sequence such that $p_{j} \downarrow 0$, and a subset $F=\left\{j_{1}<j_{2}<\ldots\right\}$ of $\mathbb{N}$, thus $\delta(F)=1$.

So we put in writing,

$$
\dot{\mu}\left(\left|x_{j}-x_{j_{i}}\right|,\left|x_{j}-x_{0}\right|+\left|x_{j_{t}}-x_{0}\right|\right)>\frac{1}{2},
$$

and,

$$
\dot{\mu}\left(\left|x_{j}-x_{0}\right|+\left|x_{j_{t}}-x_{0}\right|, 2 p_{j}\right)>\frac{1}{2}, \quad \forall \mathrm{i}, \mathrm{j} \in \mathbb{N} .
$$

As a result by transitivity,

$$
\dot{\mu}\left(\left|x_{j}-x_{j_{t}}\right|, 2 p_{j}\right)>\frac{1}{2}, \quad \forall \mathrm{i}, \mathrm{j} \in \mathbb{N} .
$$

Since a sequence $\left(x_{j}\right)_{j \in \mathbb{N}}$ is Fso- convergent and $\left(x_{j_{i}}\right)_{i \in \mathbb{N}}$ is fuzzy convergent, implies that $\delta\left(\left\{j \in \mathbb{N}: \dot{\mu}\left(2 p_{j},\left|x_{j}-x_{j_{t}}\right|\right)>\frac{1}{2}\right\}\right)=0$.

Next suppose for $\left(p_{j}\right)_{j \in \mathbb{N}}$ be a O-sequence moreover a subset $F=\left\{j_{1}<j_{2}<\ldots\right\}$ of $\mathbb{N}$ such that $\delta(F)=1$ and a 


\section{Al-Nahrain Journal of Science}

ANJS, Vol.23 (3), September, 2020, pp. $61-67$

subsequence $\left(x_{j_{i}}\right)_{i \in \mathbb{N}}$ of $\left(x_{j}\right)_{j \in \mathbb{N}}$ such that $\left(x_{j_{i}}\right)_{i \in \mathbb{N}}$ be
fuzzy order convergent to $x_{0} \in X$ and
$\delta\left(\left\{j \in \mathbb{N}: \dot{\mu}\left(p_{j},\left|x_{j}-x_{j_{i}}\right|\right)>\frac{1}{2}\right\}\right)=0$.

Additionally for every $i \in \mathbb{N}$,

$$
\dot{\mu}\left(\left|x_{j}-x_{0}\right|,\left|x_{j}-x_{j_{i}}\right|+\left|x_{j_{i}}-x_{0}\right|\right)>\frac{1}{2},
$$

and

$$
\dot{\mu}\left(\left|x_{j}-x_{j_{t}}\right|+\left|x_{j_{t}}-x_{0}\right|, 2 p_{j}\right)>\frac{1}{2},
$$

i.e.,

$$
\begin{aligned}
& \left\{j \in \mathbb{N}: \dot{\mu}\left(p_{j},\left|x_{j}-x_{0}\right|\right)>\frac{1}{2}\right\} \subseteq \\
& \left\{j \in \mathbb{N}: \dot{\mu}\left(p_{j},\left|x_{j}-x_{j_{t}}\right|\right)>\frac{1}{2}\right\} \\
& \cup\left\{j \in \mathbb{N}: \dot{\mu}\left(p_{j},\left|x_{j_{t}}-x_{0}\right|\right)>\frac{1}{2}\right\}
\end{aligned}
$$

thereby, for every $i \in \mathbb{N}$

$$
\begin{aligned}
& \delta\left(\left\{j \in \mathbb{N}: \dot{\mu}\left(p_{j},\left|x_{j}-x_{0}\right|\right)>\frac{1}{2}\right\}\right) \leq \\
& \delta\left(\left\{j \in \mathbb{N}: \dot{\mu}\left(p_{j},\left|x_{j}-x_{j_{t}}\right|\right)>\frac{1}{2}\right\}\right) \\
& +\delta\left(\left\{j \in \mathbb{N}: \dot{\mu}\left(p_{j},\left|x_{j_{t}}-x_{0}\right|\right)>\frac{1}{2}\right\}\right) .
\end{aligned}
$$

for that reason the set, $\left\{j \in \mathbb{N}: \dot{\mu}\left(p_{j},\left|x_{j_{t}}-x_{0}\right|\right)>\frac{1}{2}\right\}$ has natural density zero. This complete the Proof of this theorem.

\section{References.}

[1] Luxemburg, W. A. J. and Zaanen. A. C., Riesz space. Vol. I, North- Holland, Amsterdam, 1971.

[2] Vuikh, B. Z., Introduction to the theory of partially ordered spaces. Wolters - Noordhoff, Ltd., Groningen, 1967.

[3] Fast, H., Sur la convergence statistique. colloquium Mathematicum, Vol. 2, pp. 241-244, 1951.

[4] Steinhaus, H., Sur la convergence ordinate et la convergence esymptotique. Colloquium Mathematicum, Vol. 2, pp.73-84, 1951.

[5] Maio G. D., Kočinac L. D. R., Statistical convergence in topology. Topologyand its Applications. 156: 28-45, 2008.

[6] Li K., Lin S., Ge Y., On statistical convergence in con metric spaces. Topology and its Applications. 196: 641-651, 2015.

[7] Aytar S., Mammadov M. A., Pehlivan S., Statistical limit inferior andlimit superior for sequences of fuzzy numbers. Fuzzy sets and systems.157:976-985, 2006.

[8] Gong Z., Zhang L., Zhu X., The statistical convergence for sequences of fuzzy-number-valued functions. Information Sciences. 295:182-195, 2015.
[9] [9] Gregori V., Minãna J. -J., Std-convergence in fuzzy metric spaces. Fuzzy Sets and Systems. 267: 140-143, 2015.

[10] Șenḉimen C., Pehlivan S., Statistical convergence in fuzzy normed linearspaces. Fuzzy Sets and Systems. 159: 361-370, 2008.

[11] Back, R. C., Generalized asymptotic density. American Journal of Mathematics, Vol. 75, pp. 335346, 1953.

[12] Šalàt, T., On statistical convergence of real numbers. Mathematica slovaca, Vol. 30, pp. 139-150, 1980.

[13] Schoenberg, I. J., The integrability of certain functions and related summability methods. The American. Mathematical Monthly, Vol. 66, pp. 361375, 1959.

[14] Katsaras A. K., Fuzzy topological vector spaces II. Fuzzy sets and systems.12:143-154, 1984.

[15] Das N. R., Das P., Fuzzy topology generated by fuzzy norm. Fuzzy Sets and Systems. 107: 349-354, 1999.

[16] Xiao J., Zhu X., On linearly topological structure and property of fuzzy normed linear space. Fuzzy Sets and Systems. 125: 153-161, 2002.

[17] Felbin C., Finite dimensional fuzzy normed linear space. Fuzzy sets and systems. 48: 239-248, 1992.

[18] Kaleva O., Seikkala S., On fuzzy metric spaces. Fuzzy Sets and Systems. 12:215-229, 1984.

[19] Fang J. -X., A note on the completions of fuzzy metric spaces and fuzzy normed spaces. Fuzzy Sets and Systems. 133: 399-407, 2002.

[20] Beg, I. and Islam, M. U., Fuzzy ordered linear spaces. Vol. 3, No. 3, 659-670, 1995.

[21] Beg, I. and Islam, M. U., Fuzzy Riesz spaces. The Journal of Fuzzy Mathematics, Vol. 2, No. 1, 211$241,1994$.

[22] Labuschagne C. C. A., Pinchuck A. L., The set of fuzzy points of a fuzzy vector lattice is not a vector lattice. Fuzzy sets and systems. 157:2783-2785, 2006.

[23] Xue X., Tao J., Statistical order convergence and statistically relatively uniform convergence in Riesz spaces. Journal of Function Spaces. 4:1-9, 2018.

[24] Venugo Alan, P., Fuzzy ordered sets, Fuzzy sets and systems. 46, 221- 226, 1992. 